\title{
Secant Degeneracy Index of the Standard Strata in The Space of Binary Forms
}

\author{
Gleb Nenashev $^{1} \cdot$ Boris Shapiro $^{1}$ (D) \\ Michael Shapiro ${ }^{2}$
}

(C) The Author(s) 2017. This article is an open access publication

\begin{abstract}
The space $P o l_{d} \simeq \mathbb{C} P^{d}$ of all complex-valued binary forms of degree $d$ (considered up to a constant factor) has a standard stratification, each stratum of which contains all forms whose set of multiplicities of their distinct roots is given by a fixed partition $\mu \vdash d$. For each such stratum $S_{\mu}$, we introduce its secant degeneracy index $\ell_{\mu}$ which is the minimal number of projectively dependent pairwise distinct points on $S_{\mu}$, i.e., points whose projective span has dimension smaller than $\ell_{\mu}-1$. In what follows, we discuss the secant degeneracy index $\ell_{\mu}$ and the secant degeneracy index $\ell_{\bar{\mu}}$ of the closure $\bar{S}_{\mu}$.
\end{abstract}

Keywords Strata of the classical discriminant $\cdot$ Secant varieties $\cdot$ Partitions

Mathematics Subject Classification Primary 14M99; Secondary 11E25 - 11P05

\section{Introduction}

Below by a form we will always mean a binary form. The standard stratification of the $d$-dimensional projective space $\mathrm{Pol}_{d}$ of all complex-valued binary forms of degree $d$ (considered up to a non-vanishing constant factor) according to the multiplicities of

\footnotetext{
$凶$ Boris Shapiro

shapiro@math.su.se

Gleb Nenashev

nenashev@math.su.se

Michael Shapiro

mshapiro@math.msu.edu

1 Department of Mathematics, Stockholm University, 10691 Stockholm, Sweden

2 Department of Mathematics, Michigan State University, East Lansing, MI 48824-1027, USA
} 
their distinct roots is a well-known and widely used construction in mathematics (see e.g. Arnol'd 2014; Vassiliev 1992; Khesin and Shapiro 1992). Its strata denoted by $S_{\mu}$ are enumerated by partitions $\mu \vdash d$. In particular, cohomology of $S_{\mu}$ with different coefficients appears in many topological problems and was intensively studied over the years, see e.g. Vassiliev (1992) and references therein.

Definition 1 Given a positive-dimensional quasi-projective variety $V \subset \mathbb{C} P^{d}$, we define its secant degeneracy index $\ell_{V}$ as the minimal positive integer $\ell$ such that there exist $\ell$ distinct points on $V$ which are projectively dependent, i.e. whose projective span has dimension at most $\ell-2$. (Observe that singular points of $V$ are not considered as collapsing distinct points. For example, according to our definition, a point of selfintersection of $V$ is still considered a just one point of $V$ ).

Remark 1 The secant degeneracy index in much more general context has been introduced by e.g. Beltrametti and Sommese (1995) while discussing the $\ell$-ampleness of the linear system of hyperplane sections for different $\ell$. This notion was further developed in a recent paper (Chiantini and Ciliberto 2010). (We have to mention that unlike many modern authors, we only consider reduced finite subschemes of $V$ ). As was pointed out to us by the anonymous referee, the secant degeneracy index has already appeared in a number of topics in algebraic geometry and, in particular, is closely connected with the identifiability of tensors and higher order normality. For example, a related question about the uniqueness of representation of generic forms of subgeneric rank as sums of powers of linear forms has been studied in a recent article (Chiantini et al. 2015).

Remark 2 Obviously, $3 \leq \ell_{V} \leq d+2$. The upper bound is attained for a rational normal curve in $\mathbb{C} P^{d}$. On the other hand, if $V$ contains $\mathbb{C} P^{1} \backslash\{$ finite set $\}$, then $\ell_{V}=3$. We owe to the anonymous referee the important observation that the above trivial upper bound $\ell_{V}=d+2$ is attained only on (Zariski open subsets of) rational normal curves and this bound can be improved as follows. Namely, if $c:=\operatorname{codim} V=d-\operatorname{dim} V$, then $\ell_{V} \leq c+2$ unless $V$ is a variety of minimal degree which in our notation means that $\operatorname{deg} V=c+1$. By the classical results of Del Pezzo and Bertini, every positivedimensional variety of minimal degree contains a line which implies that $\ell_{V}=3$, unless $V$ is a rational normal curve or a Veronese surface, see e.g. Theorem 1 of Eisenbud and Harris (1987). For the Veronese surface (which is a surface in $\mathbb{C} P^{5}$ ), $\ell_{V}=4=c+1$. To see that, one can take 4 points on a conic. Thus, one concludes that $\ell_{V} \leq c+2$ unless $V$ is a Zariski open subset of a rational normal curve in which case $\ell_{V}=c+3=d+2$.

Observe that if a quasi-projective variety $V$ is contained in quasi-projective $W$, then $\ell_{V} \geq \ell_{W}$. For a positive-dimensional quasi-projective variety $V \subset \mathbb{C} P^{d}$, denote by $\ell_{\bar{V}}$ the secant degeneracy index of the closure $\bar{V} \subset \mathbb{C} P^{d}$. Obviously, $\ell_{\bar{V}} \leq \ell_{V}$. The latter inequality can be strict as shown by Example 1 below.

The principal question considered in the present paper is as follows.

Problem 1 For a given partition $\mu \vdash d$, calculatelestimate its secant degeneracy indices $\ell_{\mu}:=\ell_{S_{\mu}}$ and $\ell_{\bar{\mu}}:=\ell_{\bar{S}_{\mu}}$. 
Example 1 For $\mu(d)=(2 d+1, d, d, d, d)$ and $\mu^{\prime}(d)=(2 d+1,2 d, 2 d), \ell_{\bar{\mu}(d)}=$ $\ell_{\mu^{\prime}(d)}=3$, but $\ell_{\mu(d)}$ grows to infinity when $d \rightarrow \infty$. (This result follows from Theorem 1 below.)

For a given partition $\mu$, the equation

$$
f_{1}+f_{2}+\cdots+f_{\ell_{\mu}}=0
$$

is called the minimal secant degeneracy relation for $S_{\mu}$. A solution of the latter equation is a collection of pairwise non-proportional forms from $S_{\mu}$ satisfying (1).

Analogously, for a given partition $\mu$, the equation

$$
f_{1}+f_{2}+\cdots+f_{\ell_{\bar{\mu}}}=0
$$

is called the minimal secant degeneracy relation for $\bar{S}_{\mu}$. A solution of the latter equation is a collection of pairwise non-proportional forms from $\bar{S}_{\mu}$ satisfying (2).

Most of our results deal with the secant degeneracy index $\ell_{\mu}$. However, the second part of Theorem 1 provides a non-trivial lower bound for $\ell_{\bar{\mu}}$ generalizing a similar result of a well-known paper Newman and Slater (1979) from 1979 where the special case of partitions with equal parts was considered.

The first result of this note is as follows. Recall the notion of the refinement partial order " $\succ$ " on the set of all partitions of a given positive integer $d$. Namely, $\mu^{\prime} \succ \mu$ in this order if $\mu^{\prime}$ is obtained from $\mu$ by merging of some parts of $\mu$. The unique minimal element of this partial order is $(1)^{d}$, while its unique maximal element is $(d)$.

For a partition $\mu=\left(\mu_{1} \geq \mu_{2} \geq \cdots \geq \mu_{r}\right)$, define its jump multiset $J_{\mu}$ as the multiset of all positive numbers in the set $\left\{\mu_{1}-\mu_{2}, \ldots, \mu_{r-1}-\mu_{r}, \mu_{r}\right\}$. We denote by $h_{\mu}$ the minimal (positive) jump of $\mu$, i.e. the minimal element of $J_{\mu}$, and by $h_{\bar{\mu}}$ the minimal jump of all partitions $\mu^{\prime} \succeq \mu$.

Theorem 1 For any $\mu=\left(\mu_{1} \geq \mu_{2} \geq \cdots \geq \mu_{r}\right)$,

$$
\ell_{\mu}>\sqrt{h_{\mu}+\frac{1}{4}}+\frac{3}{2} \text {, }
$$

and

$$
\text { (ii) } \ell_{\bar{\mu}}>\sqrt{h_{\bar{\mu}}+\frac{1}{4}}+\frac{3}{2} \text {. }
$$

To formulate further results, we divide the set of all partitions into two natural disjoint subclasses as follows.

Notation. For a given partition $\mu=\left(\mu_{1} \geq \mu_{2} \geq \cdots \geq \mu_{r}\right)$ and a non-negative integer $t$, define the partition $\mu^{\langle t\rangle}$ as

$$
\mu^{\langle t\rangle}:=\left(\mu_{1}+t \geq \mu_{2}+t \geq \cdots \geq \mu_{r}+t\right) .
$$

Definition 2 We say that a partition $\mu$ has a growing secant degeneracy index if $\lim _{t \rightarrow \infty} \ell_{\mu^{\langle t\rangle}}=+\infty$ and we say that $\mu$ has a stabilising secant degeneracy index otherwise. 
We are able to characterize these two classes in the following terms.

Definition 3 Given a partition $\mu$ and a positive integer $m$, a solution of

$$
f_{1}+f_{2}+\cdots+f_{m}=0
$$

with pairwise non-proportional $f_{i} \in S_{\mu}$ is called a common radical solution if all $f_{i}$ 's have the same radical, i.e. the same set of distinct linear factors (considered up to a constant factor). We call a partition $\mu$ such that there exists $m$ and a common radical solution of (3) a partition admitting a common radical solution.

The following proposition is straightforward.

Proposition 2 A partition $\mu=\left(\mu_{1} \geq \mu_{2} \geq \cdots \geq \mu_{r}\right)=\left(i_{1}^{m_{1}}, i_{2}^{m_{2}}, \ldots, i_{s}^{m_{s}}\right)$ with distinct $i_{j}$ 's has a stabilising secant degeneracy index if and only if, for some positive integer $m$, there exists a common radical solution of (3). A partition $\mu$ as above has a growing secant degeneracy index if and only if the linear span of the Sym $m_{r}$-orbit of any form $f \in S_{\mu}$ has the dimension equal to the multinomial coefficient $\frac{r !}{m_{1} ! m_{2} ! \ldots m_{s} !}$. (Here the symmetric group Sym $m_{r}$ acts on any $f \in S_{\mu}$ by permuting all its $r$ distinct roots.)

At the moment we do not have a purely combinatorial description of partitions admitting a common radical solution. However we were able to study a somewhat stronger property.

Definition 4 We say that a partition $\mu=\left(\mu_{1} \geq \mu_{2} \geq \cdots \geq \mu_{r}\right)$ admits a strongly common radical solution if for some integer $m$, a common radical solution exists for any choice of $r$ distinct roots.

Theorem 3 A partition $\mu=\left(\mu_{1} \geq \mu_{2} \geq \cdots \geq \mu_{r}\right)$ admits a strongly common radical solution if there exists a sequence $\left\{a_{1}, \ldots, a_{r}\right\}$ of positive integers such the number of different permutations $\pi$ of $\mu$ such that $(\pi \circ \mu)_{i} \geq a_{i}$ is at least $|\mu|-$ $\sum_{i=1}^{r} a_{i}+2$, where $(\pi \circ \mu)_{i}$ is the $i$-th entry of the partition $(\pi \circ \mu)$.

In fact, we strongly suspect that the converse to Theorem 3 holds as well.

Conjecture 1 A necessary and sufficient condition for a partition $\mu=\left(\mu_{1} \geq \mu_{2} \geq\right.$ $\cdots \geq \mu_{r}$ ) to admit a strongly common radical solution is given by the existence of a sequence $\left\{a_{1}, \ldots, a_{r}\right\}$ of positive integers such the number of different permutations $\pi$ of $\mu$ such that $(\pi \circ \mu)_{i} \geq a_{i}$ is at least $|\mu|-\sum_{i=1}^{r} a_{i}+2$, where $(\pi \circ \mu)_{i}$ is the $i$-th entry of the partition $(\pi \circ \mu)$.

At the moment we can settle Conjecture 1 for a large class of partitions, but not for all partitions.

The structure of the paper is as follows. In Sect. 2, we formulate several general results about $\ell_{\mu}$, the most interesting of them being an upper bound of $\ell_{\mu}$ in terms of the minimal jump. In Sect. 3, we discuss common radical solutions of (1) and in Sect. 4 , we present a number of open problems. 


\section{General Results on the Secant Degeneracy Index}

Given a partition $\mu=\left(\mu_{1} \geq \mu_{2} \geq \cdots \geq \mu_{r}\right)$, we call $v=\left(\mu_{i_{1}} \geq \mu_{i_{2}} \geq \cdots \geq \mu_{i_{s}}\right)$ where $1 \leq i_{1}<i_{2}<\cdots<i_{s} \leq r$, a subpartition of $\mu$.

Proposition 4 For a partition $\mu=\left(\mu_{1} \geq \mu_{2} \geq \cdots \geq \mu_{r}\right)$ and any subpartition $v$ of $\mu$, the inequality

$$
\ell_{\mu} \leq \ell_{\nu}
$$

holds. In particular, $\ell_{\mu} \leq \mu_{r}+2$.

Proof Given a subpartition $v=\left(\mu_{i_{1}} \geq \mu_{i_{2}} \geq \cdots \geq \mu_{i_{s}}\right)$ of a partition $\mu=\left(\mu_{1} \geq\right.$ $\left.\mu_{2} \geq \cdots \geq \mu_{r}\right)$, let

$$
f_{1}+f_{2}+\cdots+f_{\ell_{v}}=0
$$

be a linear dependence of pairwise non-proportional binary forms from $S_{v}$ realizing its secant degeneracy index. Take the partition $\widehat{\mu}=\mu \backslash v=\left(\widehat{\mu}_{1} \geq \widehat{\mu}_{2} \geq \cdots \geq \widehat{\mu}_{r-s}\right)$. Multiplying the latter equality by $\prod_{j=1}^{r-s}\left(x-a_{j} y\right)^{\widehat{\mu}_{j}}$, where $a_{j}$ are generic complex numbers, we get a linear dependence between polynomials in $S_{\mu}$. The inequality $\ell_{\mu} \leq \mu_{r}+2$ is a special case of the general inequality, if one chooses $v=\left(\mu_{r}\right)$. Observe that for the partition $(d) \vdash d, \ell_{(d)}=d+2$, since the set of binary forms of degree $d$ with a root of multiplicity $d$ is a rational normal curve in ool $_{d} \simeq \mathbb{C} P^{d}$.

Example 2 The latter upper bound $\ell_{\mu} \leq \mu_{r}+2$ is sharp in case of any partition with $\mu_{r}=1$, but not in general. Namely, already for $\mu=\left(2^{2}\right) \vdash 4, \ell_{\mu}=3<4=\mu_{2}+2$. For $\mu=\left(3^{2}\right) \vdash 6, \ell_{\mu}=4<5 ; \mu=\left(4^{2}\right) \vdash 8, \ell_{\mu}=4<6$, see Reznick (1999).

Before formulating general results about $\ell_{\mu}$, let us present several concrete classes of $\mu$ and some information about the corresponding $\ell_{\mu}$.

Proposition 5 Let $\mu=\left(\mu_{1} \geq \mu_{2} \geq \cdots \geq \mu_{r}\right)$ be a partition with two different indices $i_{1}$ and $i_{2}$ such that $\mu_{i_{1}}-\mu_{i_{1}+1}=\mu_{i_{2}}-\mu_{i_{2}+1}=1$. Then, $\ell_{\mu} \leq 4$.

Proof Without loss of generality, assume that $i_{1}<i_{2}$, and consider two different cases.

Case $1 . i_{2}=i_{1}+1$. Take a subpartition $v=\left(\mu_{i_{1}}, \mu_{i_{1}+1}, \mu_{i_{1}+2}\right)=\left(\mu_{i_{1}+2}+2, \mu_{i_{1}+2}+\right.$ $\left.1, \mu_{i_{1}+2}\right)$ and set $k=\mu_{i_{1}+2}$. We know that $\ell_{\mu} \leq \ell_{\nu}$. So it is enough to prove that $\ell_{v} \leq 4$. Take three distinct complex numbers $p, q$ and $r$, and consider four polynomials

$$
\begin{array}{cc}
g_{1}=(x-p)^{k+2}(x-q)^{k+1}(x-r)^{k}, & g_{2}=(x-p)^{k+2}(x-r)^{k+1}(x-q)^{k}, \\
g_{3}=(x-q)^{k+2}(x-p)^{k+1}(x-r)^{k}, & g_{4}=(x-r)^{k+2}(x-p)^{k+1}(x-q)^{k} .
\end{array}
$$

A linear combination $a g_{1}+b g_{2}+c g_{3}+d g_{4}$ is given by

$$
Q(x)\left(a(x-p)(x-q)+b(x-p)(x-r)+c(x-q)^{2}+d(x-r)^{2}\right),
$$


where $Q(x)=(x-p)^{k+1}(x-q)^{k}(x-r)^{k}$. Polynomials $(x-p)(x-q),(x-p)(x-r)$, $(x-q)^{2}$ and $(x-r)^{2}$ are linearly dependent. Thus there exist $a, b, c, d$ such that $a g_{1}+b g_{2}+c g_{3}+d g_{4}=0$. Hence $\ell_{v} \leq 4$.

Case 2. $i_{2}>i_{1}+1$. Take a subpartition $v=\left(\mu_{i_{1}}, \mu_{i_{1}+1}, \mu_{i_{2}}, \mu_{i_{2}+1}\right)=\left(\mu_{i_{1}+1}+\right.$ $\left.1, \mu_{i_{1}+1}, \mu_{i_{2}+1}+1, \mu_{i_{2}+1}\right)$; set $k_{1}=\mu_{i_{1}+1}$ and $k_{2}=\mu_{i_{2}+1}$. We know that $\ell_{\mu} \leq \ell_{\nu}$. So it is enough to prove that $\ell_{v} \leq 4$. Take four distinct complex numbers $p, q, r$ and $t$, and consider four polynomials

$$
\begin{gathered}
g_{1}=(x-p)^{k_{1}+1}(x-q)^{k-1}(x-r)^{k_{2}+1}(x-s)^{k_{2}}, \\
g_{2}=(x-q)^{k_{1}+1}(x-p)^{k-1}(x-r)^{k_{2}+1}(x-s)^{k_{2}}, \\
g_{3}=(x-p)^{k_{1}+1}(x-q)^{k-1}(x-s)^{k_{2}+1}(x-r)^{k_{2}}, \\
g_{4}=(x-q)^{k_{1}+1}(x-p)^{k-1}(x-s)^{k_{2}+1}(x-r)^{k_{2}} .
\end{gathered}
$$

A linear combination $a g_{1}+b g_{2}+c g_{3}+d g_{4}$ is given by

$R(x)(a(x-p)(x-r)+b(x-q)(x-r)+c(x-p)(x-s)+d(x-q)(x-s))$,

where $R(x)=(x-p)^{k_{1}}(x-q)^{k_{1}}(x-r)^{k_{2}}(x-s)^{k_{2}}$. Polynomials $(x-p)(x-r)$, $(x-q)(x-r),(x-p)(x-s)$ and $(x-q)(x-s)$ are linearly dependent. Thus there exist $a, b, c, d$ such that $a g_{1}+b g_{2}+c g_{3}+d g_{4}=0$, and hence $\ell_{v} \leq 4$.

Definition 5 By the radical of a given binary form we mean the binary form obtained as the product of all distinct linear factors of the original form.

Proposition 6 For any partition $\mu=\left(\mu_{1} \geq \mu_{2} \geq \cdots \geq \mu_{r}\right)$ and given an arbitrary positive integer $i$, consider the partition $\mu^{\prime}=\left(\mu_{1}+i \geq \mu_{2}+i \geq \cdots \geq \mu_{r}+i \geq\right.$ $i, i, \ldots, i)$, where the entry $i$ is repeated $r\left(\ell_{\mu}-1\right)$ times at the end of $\mu^{\prime}$. Then, $\ell_{\mu^{\prime}} \leq \ell_{\mu}$.

Proof Let $f_{1}, \ldots, f_{\ell_{\mu}}$ be a solution of (1). Consider the radical $g$ of the polynomial $f_{1} f_{2} \ldots f_{\ell_{\mu}}$. Since every form $f_{j}$ has exactly $r$ distinct roots, the degree of $g$ is less than $r \ell_{\mu}$.

Construct $g^{\prime}$ as the product of $g$ by $r \ell_{\mu}-\operatorname{deg}(g)$ new distinct linear forms, and set $f_{j}^{\prime}=f_{j} \cdot\left(g^{\prime}\right)^{i}$, for $j=1, \ldots, \ell_{\mu}$. It is easy to see that each $f_{j}^{\prime}$ has the root partition given by $\mu^{\prime}$. Furthermore, one has

$$
f_{1}^{\prime}+\cdots+f_{\ell_{\mu}}^{\prime}=\left(f_{1}+\cdots+f_{\ell_{\mu}}\right) \cdot\left(g^{\prime}\right)^{i}=0
$$

hence, $\ell_{\mu^{\prime}} \leq \ell_{\mu}$.

Corollary 1 For any partition $\mu$ containing the subpartition $v=(t+1, t, t)$, where $t$ is a positive integer, the secant degeneracy index $\ell_{\mu}$ equals 3. More generally, for any positive integer $t$, and any partition $\mu$ containing the subpartition $v=(t+$ $i, \underbrace{t, t, \ldots, t}_{i+1})$, the secant degeneracy index $\ell_{\mu}$ is at most $i+2$. 
We finish this section with the proof of Theorem 1.

Proof Both parts of Theorem 1 are settled in a similar way. Namely, given $\mu$, let $\left\{f_{1}, \ldots, f_{\ell}\right\}$ be a collection of forms solving either (1) or (2). (In the first case $\ell=\ell_{\mu}$ and in the second case $\ell=\ell_{\bar{\mu}}$.) Assume that $\left\{f_{1}, \ldots, f_{\ell}\right\}$ gives a counterexample to the statement. Denote by $g$ the GCD of $\left\{f_{1}, \ldots, f_{\ell}\right\}$ and consider the relation

$$
\frac{f_{1}}{g}+\cdots+\frac{f_{\ell}}{g}=0
$$

In case (i) of Theorem 1 , for any $i$, every root of the polynomial $\frac{f_{i}}{g}$ has multiplicity at least $h_{\mu}$, because this multiplicity equals $\mu_{k}-\mu_{l}$ for some $k \leq l$. Observe that, for all $k \leq l, \mu_{k}-\mu_{l}$ is either 0 or is greater than or equal to $h_{\mu}$.

In case (ii) of Theorem 1 , for any $i$, every root of the polynomial $\frac{f_{i}}{g}$ has multiplicity at least $h_{\bar{\mu}}$, because this multiplicity equals $\sum_{k \in A} \mu_{k}-\sum_{l \in B} \mu_{l}$, where $A$ and $B$ are two subsets of $\{1, \ldots, r\}$. Observe that, for all pairs $\left(A^{\prime}, B^{\prime}\right)$ such that $A^{\prime} \cap B^{\prime}=\emptyset$, $\left|\sum_{k \in A^{\prime}} \mu_{k}-\sum_{l \in B^{\prime}} \mu_{l}\right|$ is either 0 or is greater than or equal to $h_{\bar{\mu}}$.

The rest of the proof is the same in both cases. In what follows, $h$ stands for $h_{\mu}$ in case (i) and for $h_{\bar{\mu}}$ in case (ii). Consider the sequence of Wronskians

$$
w_{i}=W\left(\frac{f_{1}}{g}, \ldots, \frac{f_{i-1}}{g}, \frac{f_{i+1}}{g}, \ldots, \frac{f_{\ell}}{g}\right), i=1, \ldots, \ell .
$$

All these Wronskians are proportional to each other due to the latter relation.

Let $\alpha$ be a root of some $f_{i}$. There exists an index $s$ such that $\frac{f_{s}}{g}$ is not divisible by $(x-\alpha)$, since otherwise $g$ is not the GCD.

For a given $t$, consider the multiplicity of the root of $w_{t}$ at $\alpha$. It satisfies the inequality:

$$
\operatorname{ord}_{\alpha}\left(w_{t}\right) \geq \sum\left(\operatorname{ord}_{\alpha}\left(\frac{f_{j}}{g}\right)\right)-(\ell-2) \#\left\{i:(x-\alpha) \mid \frac{f_{i}}{g}\right\}
$$

because any column of the Wronski matrix corresponding to $(x-\alpha) \mid \frac{f_{j}}{g}$ is divisible by $(x-\alpha)^{\operatorname{ord}_{\alpha}\left(\frac{f_{j}}{g}\right)-\ell+2}$.

Hence,

$$
\begin{aligned}
\operatorname{deg} w_{1} & \geq \sum_{i=1}^{\ell}\left(\operatorname{deg}\left(\frac{f_{i}}{g}\right)-(\ell-2) \#_{\text {roots }}\left(\frac{f_{i}}{g}\right)\right) \\
& =\ell(|\mu|-\operatorname{deg} g)-(\ell-2) \sum_{i=1}^{\ell} \#_{\text {roots }}\left(\frac{f_{i}}{g}\right) .
\end{aligned}
$$

On the other hand,

$$
\operatorname{deg} w_{1} \leq(\ell-1)\left(\operatorname{deg}\left(\frac{f_{i}}{g}\right)-\ell+2\right)=(\ell-1)(|\mu|-\operatorname{deg} g)-(\ell-1)(\ell-2)
$$


We obtain

$(\ell-1)(|\mu|-\operatorname{deg} g)-(\ell-1)(\ell-2) \geq \ell(|\mu|-\operatorname{deg} g)-(\ell-2) \sum_{i=1}^{\ell} \#_{\text {roots }}\left(\frac{f_{i}}{g}\right)$, i.e.,

$$
(\ell-2) \sum_{i=1}^{\ell} \#_{\text {roots }}\left(\frac{f_{i}}{g}\right)-(\ell-1)(\ell-2) \geq|\mu|-\operatorname{deg} g .
$$

The number $\#_{\text {roots }}\left(\frac{f_{i}}{g}\right)$ of distinct roots is at most $\frac{|\mu|-\operatorname{deg} g}{h}$, because each root has multiplicity at least $h$. Thus

$$
(\ell-2)(\ell-1) \frac{|\mu|-\operatorname{deg} g}{h_{\mu}}-(\ell-1)(\ell-2) \geq|\mu|-\operatorname{deg} g .
$$

Hence, $(\ell-2)(\ell-1)>h$.

\section{Partitions with Growing and Stabilising Secant Degeneracy Index}

Theorem 7 If $\mu=\left(\mu_{1} \geq \mu_{2} \geq \cdots \geq \mu_{r}\right)$ satisfies the inequality

$$
m \leq \sqrt{\frac{\mu_{r}}{r-1}}+1
$$

then any solution of (3) is a common radical solution.

Proof Assume the opposite. Let $\left\{f_{1}, \ldots, f_{m}\right\}$ be a solution of (1) which is not a common radical solution. Let $g$ be the GCD of $\left\{f_{1}, \ldots, f_{m}\right\}$.

For the term $f_{i}=c_{i}\left(x-a_{i, 1}\right)^{\mu_{1}} \cdots\left(x-a_{i, r}\right)^{\mu_{r}}$, define

$$
g_{i}:=\left(x-a_{i, 1}\right)^{\mu_{1}-m+2} \cdots\left(x-a_{i, r}\right)^{\mu_{r}-m+2} .
$$

Observe that $g_{i}$ is a polynomial, because any root of $f_{i}$ has multiplicity at least $\mu_{r}>m$.

Consider the sequence of Wronskians

$$
w_{i}=W\left(f_{1}, \ldots, f_{i-1}, f_{i+1}, \cdots, f_{m}\right), i=1, \ldots, m .
$$

They are proportional to each other, because $f_{1}+\ldots+f_{m}=0$. Notice that, for $i \neq t$, the column in the Wronski matrix for $w_{t}$ corresponding to $f_{i}$ is divisible by $g_{i}$. Hence $w_{t}$ is divisible by $\prod_{i=1}^{m} g_{i} / g_{t}$.

Since $\left\{f_{1}, \ldots, f_{m}\right\}$ is not a common radical solution, there exists $\alpha \in \mathbb{C}$, such that $\alpha$ is a root of $f_{p}$ but is not a root of $f_{q}$ for some $p \neq q$.

Since the Wronskians $w_{p}$ and $w_{q}$ are proportional, they are divisible by

$$
\operatorname{LCM}\left(\frac{\prod_{i=1}^{m} g_{i}}{g_{p}}, \frac{\prod_{i=1}^{m} g_{i}}{g_{q}}\right)=\frac{\prod_{i=1}^{m} g_{i}}{G C D\left(g_{p}, g_{q}\right)}=\frac{\prod_{i=1}^{m} g_{i}}{g_{p}} \frac{g_{p}}{G C D\left(g_{p}, g_{q}\right)} .
$$


Then these Wronskians are divisible by $\frac{\prod_{i=1}^{m} g_{i}}{g_{p}}(x-\alpha)^{\mu_{r}-m+2}$. Therefore their degrees are greater than or equal to

$$
(m-1)(|\mu|-r(m-2))+\mu_{r}-m+2 .
$$

On the other hand, the degrees of the Wronskians are at most $(m-1)(|\mu|-m+2)$. Thus,

$$
(m-1)(|\mu|-m+2) \geq(m-1)(|\mu|-r(m-2))+\mu_{r}-m+2,
$$

which implies that $-(m-1)(m-2) \geq-r(m-1)(m-2)+\mu_{r}-m+2$. After straightforward simplifications the latter inequality gives

$$
m-1 \geq \sqrt{\frac{\mu_{r}}{r-1}}
$$

Contradiction.

Corollary 2 For $\mu=\left(\mu_{1} \geq \mu_{2} \geq \cdots \geq \mu_{r}\right)$, either $\ell_{\mu} \geq \sqrt{\frac{\mu_{r}}{r-1}}+1$ or any solution of (1) is a common radical solution.

Remark 3 For any partition $\mu$ with a growing secant degeneracy index, i.e., for $\ell_{\mu^{\langle t\rangle}} \rightarrow$ $\infty$, we know that

$$
\sqrt{\frac{\mu_{r}+t}{r-1}}+1 \leq \ell_{\mu^{\langle t\rangle}} \leq \mu_{r}+t+2
$$

see Proposition 4 and Theorem 1.

Now we present a sufficient condition for $\mu$ to have a growing secant degeneracy index.

Corollary 3 Any partition $\mu=\left(\mu_{1} \geq \mu_{2} \geq \cdots \geq \mu_{r}\right)$, such that every its jump is at least $(r !)^{2}$, has a growing secant degeneracy index.

Proof Assume that $\ell_{\mu^{\langle t\rangle}}$ does not grow to infinity. Then by Theorem 1,

$$
\ell_{\mu^{\langle t\rangle}} \geq \sqrt{h_{\mu}^{t}+\frac{1}{4}}+\frac{3}{2} \geq \sqrt{h_{\mu}^{0}+\frac{1}{4}}+\frac{3}{2} \geq \sqrt{(r !)^{2}+\frac{1}{4}}+\frac{3}{2}>r ! .
$$

However the number of different polynomials (up to a constant factor) with fixed $r$ roots of multiplicities $\mu^{\langle t\rangle}$ is at most $r$ !. Hence no common radical solution can exist. Contradiction.

We continue with the proof of Theorem 3 . 
Proof Let $f=\left(x-c_{1} y\right)^{\mu_{1}} \cdot\left(x-c_{2} y\right)^{\mu_{2}} \cdot \ldots \cdot\left(x-c_{r} y\right)^{\mu_{r}}$ be any form from $S_{\mu}$. Consider the set $\mathcal{D}_{\left(a_{1}, \ldots, a_{r}\right)}$ of permutations of the multiset $\tau_{\mu}$ satisfying the asumptions (i) and (ii) of Theorem 3. For any $\pi \in \mathcal{D}_{\left(a_{1}, \ldots, a_{r}\right)}$, define $f_{\pi}$ as the form from the $S y m_{r}$-orbit of $f$ corresponding to $\pi$. Any such form $f_{\pi}$ is divisible by $g=\left(x-c_{1} y\right)^{a_{1}} \cdot(x-$ $\left.c_{2} y\right)^{a_{2}} \cdot \ldots \cdot\left(x-c_{r} y\right)^{a_{r}}$, because $\pi$ satisfies the above assumptions.

For any $\pi \in \mathcal{D}_{\left(a_{1}, \ldots, a_{r}\right)}$, define $\hat{f}_{\pi}:=\frac{f_{\pi}}{g} \in S_{|\mu|-\sum_{i=1}^{r} a_{i}}$. If $\left|\mathcal{D}_{\left(a_{1}, \ldots, a_{r}\right)}\right| \geq|\mu|-$ $\sum_{i=1}^{r} a_{i}+2$, then the forms $\hat{f}_{\pi}$ are linearly dependent. Therefore, the forms $f_{\pi}$, where $\pi$ runs over $\mathcal{D}_{\left(a_{1}, \ldots, a_{r}\right)}$, are also linearly dependent.

The next proposition shows that if there are many jumps of small sizes, then the secant degeneracy index is bounded.

Proposition 8 Let $d$ be a positive integer greater than 45 . For a partition $\mu=\left(\mu_{1} \geq\right.$ $\mu_{2} \geq \cdots \geq \mu_{r}$ ), if the number of jumps of sizes less than or equal to $d$ is at least $2(\log d+\log \log d+2)$, then $\ell_{\mu} \leq d(\log d+\log \log d)+2$. (Here by $\log$ we mean the binary logarithm, i.e. the logarithm with base 2.)

Proof Assume that there are at least $2(\log d+\log \log d)+2)$ such jumps. Consider every second such jump; the number of these jumps is at least $t=[\log d+\log \log d+2]$. Assume that they occupy positions $j_{1}<\ldots<j_{t}$, i.e. $\left(\mu_{j_{i}}-\mu_{j_{i}+1}\right) \leq d$, for $i \in[1, t]$. Furthermore $j_{i}+1<j_{i+1}$, because there is a nontrivial jump between them.

Consider the set of permutations of $\mu=\left\{\mu_{1}, \ldots, \mu_{r}\right\}$ such that:

- $i \notin\left\{j_{1}, \ldots, j_{t}\right\}, \pi_{i} \geq \mu_{i}$;

- $i \in\left\{j_{1}, \ldots, j_{t}\right\}, \pi_{i} \geq \mu_{i+1}$.

The number of such permutations is $2^{t}$. By Theorem 3, there is a solution of the size $\sum_{i=1}^{t}\left(\mu_{j_{i}}-\mu_{j_{i}+1}\right)+2 \leq d \cdot t+2$, because

$$
\begin{aligned}
d \cdot t+2 & \leq d \cdot(\log d+\log \log d+2)+2 \leq d \cdot(\log d+\log \log d+3) \\
& \leq d \cdot 2 \cdot \log d \leq 2^{\log d+\log \log d+1} \leq 2^{t}
\end{aligned}
$$

which finishes the proof.

\subsection{Examples}

It is rather obvious that all partitions with two parts have growing secant degeneracy index. Indeed, if there exists a common radical solution of (3), then its length $m$ is smaller than or equal to $r$ ! which in case of two parts equals 2 .

Proposition 9 (i) For partitions $\mu=(p, 2)$, one has that $\ell_{\mu}=3$ when $p=2$, and $\ell_{\mu}=4$ when $p>2$.

(ii) For partitions $\mu=(p, 3)$, one has that $\ell_{\mu}=4$ when $p=3,4,5$ and $\ell_{\mu}=5$ when $p>7$. Cases $\mu=(6,3)$ and $\mu=(7,3)$ are still open.

Proof In case $\mu=(3,4)$ we found an example:

$$
y^{3}(x+y)^{4}-y^{3} x^{4}=L(x+a y)^{3} y^{4}+(1-L)(x+b y)^{3} y^{4},
$$


where $a=3-\sqrt{3}, b=3+\sqrt{3}$ and $L=\frac{9-5 \sqrt{3}}{18}$;

In case $\mu=(5,3)$ we found an example:

$$
f_{1}+f_{2}-f_{3}-f_{4}=0
$$

where $f_{1}(x)=\left(x+c_{1}^{5} y\right)^{3}\left(x+c_{1}^{-3} y\right)^{5} ; f_{2}(x)=\left(x+c_{2}^{5} y\right)^{3}\left(x+c_{2}^{-3} y\right)^{5} ; f_{3}(x)=$ $\left(x+c_{1}^{-5} y\right)^{3}\left(x+c_{1}^{3} y\right)^{5} ; f_{4}(x)=\left(x+c_{2}^{-5} y\right)^{3}\left(x+c_{2}^{3} y\right)^{5}$. Here

$$
c_{1}=-c_{2}=\left(\frac{1+i \sqrt{35}}{6}\right)^{\frac{1}{4}}
$$

Using computer algebra packages we were able to prove the following statement.

Proposition 10 For a partition $\mu$ with three parts $a \leq b \leq c$, the following three conditions are equivalent:

(i) $\mu$ has a stabilising secant degeneracy index;

(ii) $\mu$ has a strongly stabilising secant degeneracy index;

(iii) the triple $a \leq b \leq c$ belongs to one of the following three types: $a=b, c=a+1$; $b=a+1, c=a+2 ; b=a+1, c=a+3$.

Proof $(i) \Leftrightarrow$ (ii). Note that any triple of distinct points in $\mathbb{C} P^{1}$ can be transformed into any other triple of distinct points in $\mathbb{C} P^{1}$ by a Möbius transformation. Therefore, if a stabilising solution exists for some choice of three distinct roots, it exists for any other triple of distinct roots.

(ii $) \Leftrightarrow$ (iii). Consider the $S_{3}$-orbit of the function $f_{1}(t)=\left(t-x_{1}\right)^{a}\left(t-x_{2}\right)^{b}\left(t-x_{3}\right)^{c}$ containing 6 pairwise distinct functions $f_{j}, j=1, \ldots, 6$ in case $a<b<c$ and 3 such functions if any two of them coincide. Denote by $W$ the corresponding Wronskian of $f_{1}, \ldots, f_{6}$ (divided by the trivial factor which is a polynomial of degree 6 in $t$ ). If $\mu=(a, b, c)$ has strongly stabilising secant degeneracy index, then $W \equiv 0$ for all $t, x_{1}, x_{2}, x_{3}$. In particular, all coefficients of $W(t)$ should vanish identically. Using Maple we able to find all possible triples $(a, b, c)$ for which all coefficients of $W(t)$ are identically 0 . The solutions are presented in (iii) above.

\section{Final Remarks and Problems}

1. Conjecture 1 from the introduction is equivalent to the claim that for an appropriate choice of an $r$-tuple of distinct complex numbers, a certain matrix whose entries are polynomials in these numbers has full rank. In other words, that some maximal minor of this matrix is a non-trivial polynomial in the latter $r$-tuple which is highly plausible. Unfortunately, the structure of the matrix is rather involved and so far we are only able to handle a large number of special cases. 
2. Conditions formulated in Proposition 2 are difficult to check which motivates the following questions.

Problem 2 Give necessary and sufficient combinatorial conditions for a partition $\mu$ to have a growing/stabilizing secant degeneracy index.

Problem 3 For any partition $\mu$ with a growing secant degeneracy index, what is the leading term of the asymptotic of $\ell_{\mu^{\langle t\rangle}}$, when $t \rightarrow+\infty$ ? Does it depend on a particular choice of $\mu$ ?

3. The next statement is obvious.

Proposition 11 The number $\ell_{\bar{\mu}}$ is monotone non-decreasing in the refinement partial order. In other words, if $\mu^{\prime} \succ \mu^{\prime \prime}$, then $\ell_{\bar{\mu}^{\prime}} \geq \ell_{\bar{\mu}^{\prime \prime}}$.

Based on a substantial number of calculations, we conjecture the following.

Conjecture 2 For any partition $\mu \vdash d$, there exists $\mu^{\prime} \succeq \mu$ such that $\ell_{\bar{\mu}}=\ell_{\mu^{\prime}}$.

Acknowledgements The second author is grateful to Professor B. Reznick of UIUC for discussions of the topic. The third author wants to acknowledge the hospitality of the Mathematics Department, Stockholm University in September-October 2015. The authors want to express their gratitude to the anonymous referee whose constructive criticism allowed us to substantially improve the quality of the exposition. The third author is supported by NSF grants DMS-1362352 and DMS-1702115.

Open Access This article is distributed under the terms of the Creative Commons Attribution 4.0 International License (http://creativecommons.org/licenses/by/4.0/), which permits unrestricted use, distribution, and reproduction in any medium, provided you give appropriate credit to the original author(s) and the source, provide a link to the Creative Commons license, and indicate if changes were made.

\section{References}

Arnol'd, V.: The cohomology ring of the colored braid group. Collect. Works 2, 183-186 (2014)

Beltrametti, M.C., Sommese, A.J.: The adjunction theory of complex projective varieties. de Gruyter Expositions in Mathematics. Walter de Gruyter \& Co., Berlin (1995)

Chiantini, L., Ciliberto, C.: On the dimension of secant varieties. J. Eur. Math. Soc. (JEMS) 12(5), 1267$1291(2010)$

Chiantini, L., Ottaviani, G., Vannieuwenhoven, N.: On generic identifiability of symmetric tensors of subgeneric rank, arXiv:1504.00547v2, April (2015)

Eisenbud, D., Harris, J.: On varieties of minimal degree (a centennial account). Algebraic geometry, Bowdoin, 1985 (Brunswick, Maine, 1985), 3-13, In: Proceedings of Symposia in Pure Mathematics, 46, Part 1, American Mathematical Society, Providence, RI, (1987)

Khesin, B., Shapiro, B.: Swallowtails and Whitney umbrellas are homeomorphic. J. Alg. Geom. 1, 549-560 (1992)

Newman, D.J., Slater, M.: Waring's problem for the ring of polynomials. J. Number Theory 11(4), 477-487 (1979)

Reznick, B.: Linearly dependent powers of quadratic forms, Preliminary report: 1999-2011, slides. https:// faculty.math.illinois.edu/ reznick/11111.pdf. Accessed 2 Dec 2017

Vassiliev, V.: Complements of discriminants of smooth maps: topology and applications. Translated from the Russian by B. Goldfarb. Translations of mathematical monographs, 98. American Mathematical Society, Providence, RI, vi+208 pp(1992) 